\title{
Left atrial remodelling among Turner syndrome patients: novel insights from non-invasive 3D echocardiography
}

\author{
Mohamed Abd El Rahman ${ }^{1,2}$, Anna-Maria Jung ${ }^{3}$, Michael Zemlin ${ }^{4}$, Tilman R. Rohrer ${ }^{3}$, Rita Schuck ${ }^{5,6}$, \\ Felix Sebastian Oberhoffer, ${ }^{1,7 \#}$, Hashim Abdul-Khaliq ${ }^{1 \#}$ \\ ${ }^{1}$ Department of Pediatric Cardiology, Saarland University Hospital, Homburg (Saar), Germany; ${ }^{2}$ Department of Pediatrics and Pediatric Cardiology, \\ Cairo University, Cairo, Egypt; ${ }^{3}$ Section for Pediatric Endocrinology, Department of General Pediatrics and Neonatology, Saarland University \\ Medical Center, Homburg (Saar), Germany; ${ }^{4}$ Department of General Pediatrics and Neonatology, Saarland University Hospital, Homburg \\ (Saar), Germany; ${ }^{5}$ Department of Congenital Heart Disease, German Heart Center Berlin, Berlin, Germany; ${ }^{6}$ Department of Rheumatology, \\ Knappschaftsklinikum Saar, Puettlingen, Germany; ${ }^{7}$ Department of Pediatric Cardiology and Pediatric Intensive Care, Medical Hospital of the \\ University of Munich, Ludwig Maximilians University Munich, Munich, Germany
}

Contributions: (I) Conception and design: All authors; (II) Administrative support: All authors; (III) Provision of study materials or patients: All authors; (IV) Collection and assembly of data: All authors; (V) Data analysis and interpretation: All authors; (VI) Manuscript writing: All authors; (VII) Final approval of manuscript: All authors.

"These authors contributed equally to this work.

Correspondence to: Hashim Abdul-Khaliq, MD. Kirrberger Straße, Geb. 9, 66421 Homburg (Saar), Germany. Email: hashim.abdul-khaliq@uks.eu.

Background: To assess (I) the left atrial (LA) size, function and (II) the impact of excess weight on the LA and left ventricular (LV) performance in Turner syndrome (TS) patients.

Methods: Twenty-five TS patients without congenital heart disease (CHD) and 19 healthy, age-matched controls underwent three-dimensional echocardiography (3DE) for LA volume measurements and twodimensional speckle tracking echocardiography (2DSTE) for LA strain measurements. LV performance was measured through LV Tei-index, indexed isovolumetric contraction (ICT/ $\sqrt{R R}$ interval), indexed relaxation (IVRT/ $\sqrt{ } \mathrm{RR}$ interval) and indexed filling time (FT/ $\sqrt{\mathrm{RR}}$ interval).

Results: Compared to healthy controls, normal-weight TS patients $(\mathrm{n}=16)$ displayed significantly increased heart rate $(92.88 \pm 16.66$ vs. $76.53 \pm 15.65 \mathrm{bpm} ; \mathrm{P}=0.005)$, reduced indexed $\mathrm{LV}$ filling time $(11.67 \pm 2.55$ vs. 15.16 \pm 5.07 ; $\mathrm{P}=0.018)$, reduced 3D maximum LA volume at $\mathrm{LV}$ end systole/BSA $\left(16.74 \pm 5.00\right.$ vs. $19.89 \pm 4.32 \mathrm{~mL} / \mathrm{m}^{2}$; $\mathrm{P}=0.05)$, reduced 3D LA total emptying volume/BSA [10.04 (5.05/18.46) vs. $13.11(7.69 / 18.46) \mathrm{mL} / \mathrm{m}^{2}$; $\mathrm{P}=0.001]$ and reduced 3D LA active emptying volume/BSA [2.61 (0.1/3.82) vs. $3.44(1.64 / 6.37) \mathrm{mL} / \mathrm{m}^{2}$; $\mathrm{P}=0.006]$. Compared to normal-weight TS patients, overweight/obese TS patients $(\mathrm{n}=9)$ showed impaired LV Tei-index [0.38 (0.26/0.55) vs. 0.27 (0.07/0.41); $\mathrm{P}=0.009]$, prolonged indexed IVRT (2.04 \pm 0.72 vs. $1.30 \pm 0.64 ; \mathrm{P}=0.015)$, prolonged indexed ICT [1.96 (1.57/2.73) vs. $1.29(0.35 / 2.69) ; \mathrm{P}=0.009]$ and increased $3 \mathrm{D}$ LA active emptying volume/BSA $\left(3.38 \pm 1.21\right.$ vs. $\left.2.29 \pm 1.07 \mathrm{~mL} / \mathrm{m}^{2} ; \mathrm{P}=0.032\right)$.

Conclusions: Normal-weight TS patients with increased heart rate and reduced LV filling time display subtle LV diastolic dysfunction in the form of reduced LA reservoir and pump function. Manifested systolic and diastolic LV dysfunction among overweight TS patients is partially compensated through an increase in LA active pump function.

Keywords: Turner syndrome (TS); 3D echocardiography; 2D speckle tracking echocardiography; left atrium

Submitted May 12, 2021. Accepted for publication Nov 23, 2021; Published: 21 Feb 2022.

doi: 10.21037/qims-21-515

View this article at: https://dx.doi.org/10.21037/qims-21-515 


\section{Introduction}

Turner syndrome (TS) is a genetic condition in females characterised by the complete or partial absence of one of the two $\mathrm{X}$ chromosomes (1). TS occurs in approximately one in 2,500-3,000 female newborns (1). Subjects display an increased cardiovascular risk and are more likely to be overweight or obese (2-4). TS is also associated with impairments of vascular function (5-8). Furthermore, recent studies of our department revealed latent left ventricular (LV) systolic and diastolic dysfunctions in a cohort of TS patients, particularly in those with excess weight (9-11).

Atrial function, a crucial determinant of ventricular filling, is known to play an important role in the pathophysiology of heart failure $(12,13)$. To the best of our knowledge, data on left atrial (LA) function and its impact on LV function has not been reported yet in TS.

LA strain derived through 2D speckle tracking echocardiography (2DSTE) and LA volume assessment through three-dimensional echocardiography (3DE) are modern imaging methods to evaluate LA performance $(14,15)$.

By utilizing the above-mentioned techniques in combination with Doppler echocardiography, the objectives of the present study were two-fold: (I) to compare LA volumes and LA function between TS patients without congenital heart disease (CHD) and healthy, age-matched controls, (II) to assess the impact of excess weight on LA and LV performance within the TS cohort.

We present the following article in accordance with the STROBE reporting checklist (available at https://qims. amegroups.com/article/view/10.21037/qims-21-515/rc).

\section{Methods}

\section{Ethical approval}

This study was a retrospective analysis of prospectively collected data. This study was conducted in accordance with the Declaration of Helsinki (as revised in 2013). This study was approved by the Ethics Committee of the Ärztekammer des Saarlandes (State Chamber of Physicians of the German federal state of Saarland), Faktoreistraße 4, 66111 Saarbrücken, Germany, on March 23rd, 2018; approval statement No. 07/18. Prior written informed consent was obtained from all patients or the parents or legal guardians of patients under legal age.

\section{Study population}

Initially, 38 TS patients with a confirmed genetic diagnosis of TS were prospectively recruited for the study between November 2016 and April 2018. TS patients were selected on the basis that they were seen regularly at the departments of pediatric cardiology and pediatric endocrinology. In collaboration with the German Turner Syndrome Association (Turner-Syndrom-Vereinigung Deutschland e.V.), additional external TS patients were included into our study.

TS exclusion criteria were congenital heart disease and/ or previous heart surgery. In addition, sinus rhythm had to be present in 12-lead ECG for study inclusion. To evaluate the presence of congenital heart disease in the participating TS subjects, an echocardiographic screening was carried out before study participation. In addition, medical records were screened. According to the above-mentioned criteria, 13 TS patients were excluded from the present study. The control group consisted of healthy, age-matched volunteers as well as patients with accidental heart murmurs (e.g., due to the prevalence of a false tendon that crosses the LV without causing an obstruction) in whom cardiac pathologies had been excluded by echocardiography and electrocardiography. The data was collected prospectively and then analysed retrospectively. The presence of normal bodyweight was a prerequisite for study participation of healthy controls.

Subjects younger than 18 years were classified as normalweight, overweight or obese based on body mass index (BMI, $\mathrm{kg} / \mathrm{m}^{2}$ ) percentiles as established by KromeyerHauschild et al. (overweight $\geq 90^{\text {th }}$ percentile, obese $\geq 97^{\text {th }}$ percentile) (16). For subjects over the age of 18 years, weight classification used the following categories: normalweight for BMI $<25 \mathrm{~kg} / \mathrm{m}^{2}$, overweight for BMI $\geq 25 \mathrm{~kg} / \mathrm{m}^{2}$ but $<30 \mathrm{~kg} / \mathrm{m}^{2}$, and obese for $\mathrm{BMI} \geq 30 \mathrm{~kg} / \mathrm{m}^{2}$.

\section{Evaluation of the left ventricular function using conventional echocardiography and pulsed tissue Doppler imaging}

All subjects underwent conventional echocardiography using either a GE M4S or a GE M5S-D phased-array transducer with a Vingmed Vivid 9 ultrasound system (General Electrics Healthcare, Fairfield, CT, USA). Examination was carried out in the left lateral decubitus position. Conventional echocardiography was performed in accordance with the American Society of Echocardiography recommendations (17). Heart rate (bpm) was continuously monitored by a three-lead ECG during echocardiographic examination. Pulsed wave Doppler measurements and 
electrocardiograph recordings were assessed simultaneously in each patient. The pulsed Doppler gate size was set to $1.5 \mathrm{~mm}$ and the filter was adjusted to $100 \mathrm{~Hz}$. For optimal acquisition, the transducer and the Doppler beam were aligned as close as possible. No angle correction was made for the Doppler examination. The mitral valve inflow velocity profiles were recorded with the Doppler sample placed at the tip of the mitral valve in the four-chamber view. Mitral inflow peak velocities were recorded at early (E peak velocity, $\mathrm{cm} / \mathrm{s}$ ) and late (A peak velocity, $\mathrm{cm} / \mathrm{s}$ ) diastole. The ratio of $\mathrm{E} / \mathrm{A}$ was calculated. In addition, the $\mathrm{LV}$ filling time (time between mitral valve opening and mitral valve closure) was measured and indexed to the $\sqrt{ } R R$ interval. To determine flow velocities of the LV outflow tract, the sample volume was placed just below the aortic valve in an apical five-chamber view. All Doppler derived parameters were measured offline. The mean value of each parameter was calculated in three consecutive cardiac cycles. The Teiindex, the isovolumetric contraction (ICT) and relaxation (IVRT) time were calculated as previously described $(18,19)$. The calculated ICT and IVRT were then indexed to the $\sqrt{R R}$ interval. Using pulsed wave tissue Doppler imaging (PW-TDI), the early diastolic (E') myocardial velocities at the basal segment of the interventricular septum (IVS) were recorded. The E/E' ratio was then calculated. To improve temporal resolution, the frame rate was kept over 180 frames/s by decreasing the sector width and depth. During acquisition, special care was taken to align the ultrasound beam parallel to the target wall.

\section{Two-dimensional speckle tracking echocardiography for the assessment of left atrial strain and aortic strain}

For speckle tracking echocardiography analysis, apical 2 - and 4-chamber views images were obtained using conventional 2-dimensional gray-scale echocardiography with a stable electrocardiographic recording. Atrial strain was calculated using apical 2 - and 4-chamber views. The QRS wave was used as the cardiac cycle starting point. Special care was taken not to foreshorten the LA which allowed for a more reliable delineation of the atrial endocardial border. Three consecutive heart cycles were recorded and averaged. The frame rate was 60-80 frames/s. Analysis was performed offline by a single experienced echocardiographer using a commercially available semiautomated 2D LA AVI software (General Electrics Healthcare, Fairfield, CT, USA). The endocardial border was traced manually in apical views. A region of interest
(ROI), composed of 6 segments, was then delineated for each view. Manual adjustments of the ROI were performed and the longitudinal strain curves for each segment were generated subsequently by the software. LA longitudinal strain was defined as the average of 2- and 4-chamber longitudinal strain curves.

Three different types of LA longitudinal strain were measured: (I) the LA peak longitudinal strain (2D LA PALS), representing the positive LA strain measured at end of reservoir phase (opening of the mitral valve) and reflecting the LA reservoir function; (II) LA longitudinal conduit strain (2D LA Conduit S), reflecting the LA longitudinal strain during conduit phase (negative value) and measured as the difference of the LA strain value at the onset of atrial contraction (PreA) minus LA strain value at end systole (mitral valve opening); (III) the LA peak longitudinal contraction strain (2D LA PACS), representing the negative LA strain at maximal atrial contraction and reflecting the LA pump function (14). The aortic strain derived from $2 \mathrm{D}$ speckle echocardiography was measured as previously described in a publication of our department (8).

\section{Three-dimensional echocardiography for the assessment of left atrial volumes and left atrial strain}

Three-dimensional echocardiography (3DE) was performed from an apical four-chamber view using a 3-D matrix array transducer (Vingmed Vivid 9 ultrasound system, General Electrics Healthcare, Fairfield, CT, USA). A wide-angle acquisition "full volume" mode was used in which 6 wedgeshaped sub-volumes were acquired for 6 consecutive cardiac cycles during a single breath-hold, resulting in a study in temporal resolution of 6 frames per cardiac cycle with a minimum frame rate of $42 / \mathrm{s}$. The entire ventricular and atrial cavities were included in the $3 \mathrm{D}$ pyramidal volume. Acquisitions were stored in a DICOM format and transferred to a separate workstation for offline data analysis. The software 4D Auto LVQ (General Electrics Healthcare, Fairfield, CT, USA) was used for strain analysis. The following parameters were acquired using 3DE: $\mathrm{LV}$ end diastolic volume (LV EDV, $\mathrm{mL}$ ), LV end systolic volume (LV ESV, mL) and LV stroke volume (LV SV, mL). To ensure objective comparability between groups, parameters were analysed relative to body surface area $\left(B S A, \mathrm{~m}^{2}\right)$. BSA was calculated using the Mosteller formula (20).

To measure the LA volume and the LA deformation during the cardiac cycle, a semi-automated segmentation algorithm was used. The segmentation algorithm was 

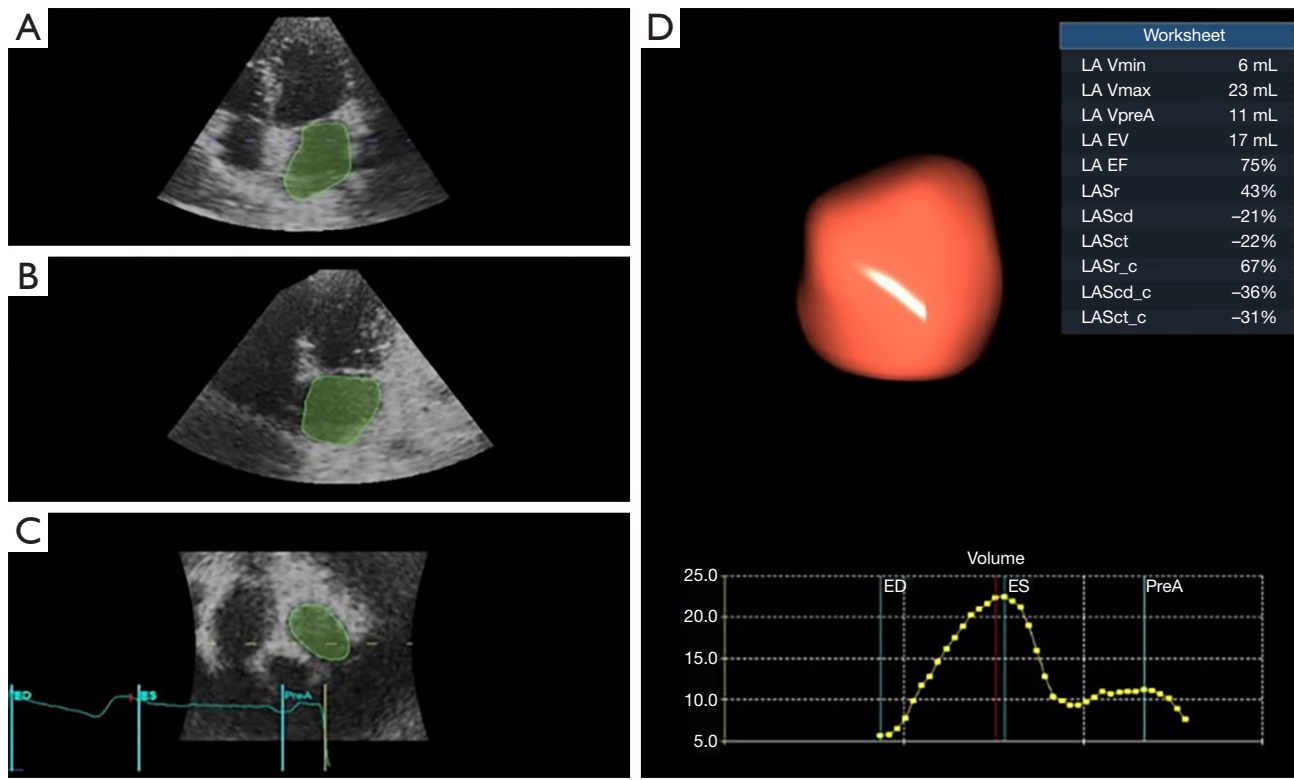

Figure 1 Three-dimensional echocardiographic assessment of left atrial (LA) volumes. LA volumes are marked in four-chamber view (A), two-chamber view (B) and in short axis (C). ES, end systolic LA volume representing the maximal LA volume; ED, end diastolic LA volume representing the minimal LA volume; PreA, LA pre-kick Volume representing the LA volume at atrial contraction. From the LA end systolic and the LA end diastolic volumes the LA total emptying volume and fraction were calculated. In addition, the software measured the following strain values: LASr, longitudinal LA strain during reservoir phase; LAScd, longitudinal LA strain during conduit phase; LASct, longitudinal LA strain during contraction phase; LASr_c, circumferential LA strain during reservoir phase; LAScd_c, circumferential LA strain during conduit phase; LASct_c, circumferential LA strain during contraction phase (D).

based on a 3D LA model and used an extended Kalman filter combining LA geometry, a motion model and edge detection algorithms. A landmark, which was placed at the center of the mitral valve annulus initialized the algorithm. The strain calculation is based on the change in length of different lines along each anatomical direction. The longitudinal strain calculation utilized eight longitudinal lines, each connecting two opposite LA basal points. The two opposite basal points were sampled from an automatically constructed triangular mesh. To calculate the circumferential strain, seven circumferential lines equidistantly distributed between the LA base and the LA apex were used. Global strain was then calculated for each direction as the average strain of the respective directional lines (21). The software subsequently measured the following LA volume values (Figure 1):

(I) Minimum LA volume at $\mathrm{LV}$ end diastole (3D LA Vmin, $\mathrm{mL}$ );

(II) Maximum LA volume at LV end systole (3D LA Vmax, mL);

(III) Volume at onset of LA contraction (3D LA VpreA, $\mathrm{mL})$.

For the assessment of the LA reservoir function, the following volumetric parameters were used:

LA total emptying volume $(m L)=L A$ Vmax $-L A$ Vmin

LA total emptying fraction $(\%)=\frac{L A \text { Vmax }-L A \text { Vmin }}{L A V \max } \times 100$

For the assessment of LA conduit function, the following volumetric parameters were used:

$L A$ passive emptying volume $(m L)=L A$ Vmax $-L A$ VpreA

LA passive emptying fraction $(\%)=\frac{L A \text { Vmax }-L A \text { VpreA }}{L A V \max } \times 100$ 
$L A$ conduit volume $(m L)=L A$ stroke volume $-($ LA Vmax $-L A$ Vmin $)$

For the assessment of LA pump function, the following volumetric parameters were used (22):

$L A$ active emptying volume $(m L)=L A$ VpreA $-L A$ Vmin

LA active emptying fraction $(\%)=\frac{L A \text { VpreA }- \text { LA Vmin }}{\text { LA VpreA }} \times 100$

The percentage contribution of each LA phase to LV SV was calculated as follows:

$L A$ reservoir contribution to $L V S V(\%)=\frac{\text { LA total emptying volume }}{L V S V} \times 100$

LA conduit contribution to $L V S V(\%)=\frac{L A \text { conduit volume }}{L V S V} \times 100$

LA passive contribution to $L V S V(\%)=\frac{L A \text { passive emptying volume }}{L V S V} \times 100$

LA pump contribution to LV SV $(\%)=\frac{L A \text { active emptying volume }}{L V S V} \times 100$

In addition, three LA longitudinal- and circumferential strain values were provided by the software:

(I) $3 \mathrm{D} \mathrm{LASr}=$ longitudinal atrial strain during reservoir phase (positive value), measured as the difference of the LA strain value at end systole (mitral valve opening) minus LA at end diastole (mitral valve closure). The reservoir phase encompasses the time of LV isovolumic contraction, ejection and isovolumic relaxation.

(II) 3D LAScd = longitudinal strain during conduit phase (negative value), measured as the difference of the LA strain value at the onset of atrial contraction (PreA) minus LA strain value at end systole (mitral valve opening).

(III) 3D LASct = longitudinal strain during contraction phase (negative value), measured as the difference of the LA strain value at end diastole (mitral valve closure) minus LA strain at onset of atrial contraction (PreA).

(IV) $3 \mathrm{D}$ LASr_c $=$ circumferential strain during reservoir phase (positive value), measured as the difference of the LA strain value at end systole (mitral valve opening) minus LA at end diastole (mitral valve closure).

(V) 3D LAScd_c = circumferential strain during conduit phase (negative value), measured as the difference of the LA strain value at the onset of atrial contraction (PreA) minus LA strain value at end systole (mitral valve opening).
(VI) 3D LASct_c $=$ circumferential strain during contraction phase (negative value), measured as the difference of the LA strain value at end diastole (mitral valve closure) minus LA strain at onset of atrial contraction (PreA).

To ensure objective comparability between groups, LA volumes were analysed relative to body surface area (BSA, $\mathrm{m}^{2}$ ). BSA was calculated using the Mosteller formula (20).

\section{Statistical analyses}

Statistical analyses were performed using SPSS 24 (Released 2016. IBM SPSS Statistics for Windows, Version 24.0. IBM Corp., Armonk, NY, USA). All continuous variables were tested for normality using the Kolmogorov-Smirnov test. Data are shown as mean \pm SD or as median, minimum and maximum if not normally distributed. Continuous variables with normal distribution were compared using the independent-samples T test. The Mann-Whitney $\mathrm{U}$ test was used to compare non-normally distributed continuous variables. Correlations were analysed using the Pearson correlation for normally distributed variables or the Spearman correlation for non-normally distributed variables. A $\mathrm{P}$ value $\leq 0.05$ was considered statistically significant. Relative intravariability was calculated for $3 \mathrm{DE}$ parameters in ten randomly selected study subjects as:

$$
\frac{|A-B|}{[(A+B) / 2]} \times 100
$$


Table 1 Characteristics of healthy controls (HC) and Turner syndrome patients (TS)

\begin{tabular}{lccc}
\hline Variables & $\mathrm{HC}(\mathrm{n}=19)$ & $\mathrm{TS}(\mathrm{n}=25)$ & $\mathrm{P}$ value \\
\hline Age (years) & $18.09 \pm 7.84$ & $14.76 \pm 5.52$ & 0.105 \\
Heart rate $(\mathrm{bpm})$ & $80(45 / 115)$ & $91(68 / 118)$ & $0.001^{\star \star *}$ \\
Height $(\mathrm{cm})$ & $164(142 / 176)$ & $148(100 / 163)$ & $0.0001^{\star \star *}$ \\
Weight $(\mathrm{kg})$ & $54.69 \pm 9.07$ & $48.23 \pm 22.01$ & 0.237 \\
BMI $\left(\mathrm{kg} / \mathrm{m}^{2}\right)$ & $20.34 \pm 2.26$ & $22.78 \pm 6.97$ & 0.151 \\
BSA $\left(\mathrm{m}^{2}\right)$ & $1.57 \pm 0.16$ & $1.35 \pm 0.38$ & $0.027^{\star}$ \\
SBP $(\mathrm{mmHg})$ & $116.68 \pm 8.24$ & $117.40 \pm 14.66$ & 0.85 \\
DBP $(\mathrm{mmHg})$ & $69.89 \pm 9.38$ & $71.32 \pm 12.21$ & 0.675 \\
\hline
\end{tabular}

BMI, body mass index; BSA, body surface area; SBP, systolic brachial blood pressure; DBP, diastolic brachial blood pressure; Mean \pm standard deviation is used for normally distributed variables and median (minimum/maximum) for non-normally distributed variables. ${ }^{*} \mathrm{P}$ value $\leq 0.05,{ }^{* \star *} \mathrm{P}$ value $\leq 0.001$

\section{Results}

\section{Patient characteristics}

Characteristics of healthy controls and TS patients are summarised in Table 1.

Out of 39 TS patients, who were prospectively recruited for this study, 13 were excluded due to morphological CHD. In addition, one boy presenting with mixed dysgenesis of the gonads was excluded from further analysis.

In total, 25 females with TS and 19 age matched controls were further analysed. Compared to controls, TS patients were significantly smaller in height and displayed significantly increased heart rate. No significant difference was seen in systolic and diastolic brachial blood pressure between TS patients and healthy peers. Four (16\%) TS patients had arterial hypertension and were prescribed angiotensin converting enzyme inhibitors. Sixteen (64\%) TS patients displayed normal-weight and nine (36\%) were overweight or obese. Thirteen (52\%) TS patients had monosomy X $(45, \mathrm{X} 0)$, while 12 (48\%) TS patients had a mosaic form of TS. Seventeen TS patients (68\%) received growth hormone replacement therapy during study participation.

\section{Evaluation of the left ventricular function using conventional echocardiography and pulsed tissue Doppler imaging}

The results of the conventional Doppler echocardiography and pulsed tissue Doppler imaging are presented in Table 2 and Table 3 for healthy controls and studied TS patients.

Compared to healthy controls, normal-weight TS patients displayed significantly reduced indexed $\mathrm{LV}$ filling time $(11.67 \pm 2.55$ vs. $15.16 \pm 5.07 ; \mathrm{P}=0.018)$. No other significant differences were observed in the remaining conventional Doppler and pulsed TDI derived parameters between healthy controls and normal-weight TS patients.

Compared to normal-weight TS patients, overweight/ obese TS patients displayed significantly higher LV Teiindex [0.38 (0.26/0.55) vs. $0.27(0.07 / 0.41) ; \mathrm{P}=0.009]$, significantly prolonged indexed ICT $[1.96(1.57 / 2.73) \mathrm{vs}$. $1.29(0.35 / 2.69) ; \mathrm{P}=0.009]$ and indexed IVRT $(2.04 \pm 0.72$ vs. $1.30 \pm 0.64 ; \mathrm{P}=0.015)$. The mid aortic abdominal strain among overweight TS patients was significantly reduced when compared to normal-weight TS patients (7.56\% $\pm 2.58 \%$ vs. $13.07 \% \pm 4.95 \% ; \mathrm{P}=0.009)$.

\section{Two-dimensional speckle tracking echocardiography for the assessment of left atrial strain}

Compared to controls, normal-weight TS patients displayed no significant difference in 2D LA PALS $(39.75 \% \pm 7.60 \%$ vs. $38.92 \% \pm 4.80 \% ; \mathrm{P}=0.698), 2 \mathrm{D}$ LA Conduit $\mathrm{S}$ $(-28.87 \% \pm 6.86 \%$ vs. $-28.97 \% \pm 4.95 \% ; \mathrm{P}=0.961)$ and $2 \mathrm{D}$ LA PACS $(-10.62 \% \pm 3.24 \%$ vs. $-9.84 \% \pm 3.73 \% ; \mathrm{P}=0.516)$. Among TS patients, 2D LA PALS correlated significantly with the $\mathrm{LV}$ E/A ratio $(\mathrm{r}=0.50 ; \mathrm{P}=0.010)$.

Regarding the following parameters, no significant difference was assessed between normal-weight and overweight/obese TS patients: $2 \mathrm{D}$ LA PALS $(39.75 \% \pm 7.60 \%$ vs. $35.44 \% \pm 10.28 \% ; \mathrm{P}=0.24), 2 \mathrm{D}$ LA Conduit S $(-28.87 \% \pm 6.86 \%$ vs. $-26.44 \% \pm 8.44 \% ; \mathrm{P}=0.44)$ and 2D LA PACS $(-10.62 \% \pm 3.24 \%$ vs. $-9.11 \% \pm 4.19 \%$; 
Table 2 Characteristics, conventional Doppler and TDI Doppler in healthy controls (HC) and normal-weight Turner syndrome patients (TS)

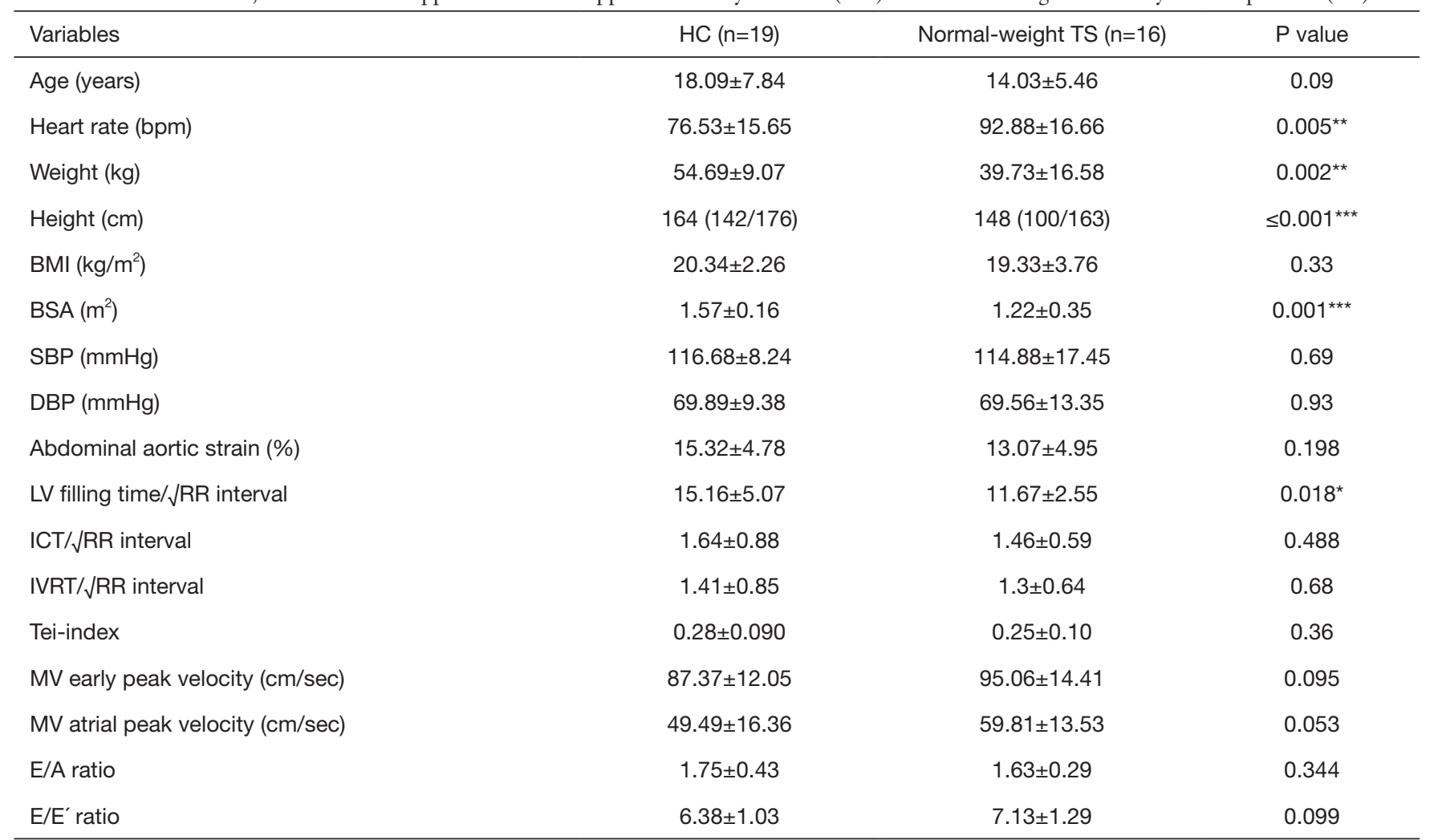

BMI, body mass index; BSA, body surface area; SBP, systolic brachial blood pressure; DBP, diastolic brachial blood pressure; ICT, isovolumetric contraction time; IVRT, isovolumetric relaxation time; E/E', pulsed MV early peak velocity to pulsed TDI (early diastolic myocardial velocities at the basal segment of the interventricular septum). Mean \pm standard deviation is used for normally distributed variables and median (minimum/maximum) for non-normally distributed variables. ${ }^{*} \mathrm{P}$ value $\leq 0.05 ;{ }^{* *} \mathrm{P}$ value $\leq 0.01$; ${ }^{* * *} \mathrm{P}$ value $\leq 0.001$.

$\mathrm{P}=0.32)$.

\section{Three-dimensional echocardiography for the assessment of left atrial volumes and left atrial strain}

The results of LA volumes and LA strain assessed using 3DE are presented in Table 4 and Table 5 for healthy controls and studied TS patients.

Compared to healthy controls, normal-weight TS patients displayed significantly reduced 3D LA Vmax/ BSA $\left(16.74 \pm 5.00 v s .19 .89 \pm 4.32 \mathrm{~mL} / \mathrm{m}^{2} ; \mathrm{P}=0.05\right), 3 \mathrm{D} \mathrm{LA}$ total emptying volume/BSA [10.04 (5.05/18.46) vs. 13.11 (7.69/18.46) $\left.\mathrm{mL} / \mathrm{m}^{2} ; \mathrm{P}=0.001\right]$ and $3 \mathrm{D}$ LA active emptying volume/BSA [2.61 (0.1/3.82) vs. $3.44(1.64 / 6.37) \mathrm{mL} / \mathrm{m}^{2}$; $\mathrm{P}=0.006$ ] (Figure 2). 3D atrial strain values showed no significant difference between healthy controls and normalweight TS patients (Table 4).

Compared to normal-weight TS patients, overweight/ obese TS patients showed a significantly higher $3 \mathrm{D}$ LA active emptying volume/BSA $(3.38 \pm 1.21 \mathrm{vs}$. $2.29 \pm 1.07 \mathrm{~mL} / \mathrm{m}^{2} ; \mathrm{P}=0.032$ ) (Figure 2). The remaining LA volume and -strain parameters assessed using $3 \mathrm{DE}$ did not show any significant differences between normal-weight and overweight/obese TS patients (Table 5).

Within the TS cohort, a significant correlation was demonstrated between heart rate and 3D LA longitudinal conduit strain $(r=0.51 ; P=0.008)$. A significant negative correlation was assessed between the LA passive contribution to LV SV and the heart rate among all studied subjects $(\mathrm{r}=-0.39 ; \mathrm{P}=0.013)$ and within the TS cohort $(\mathrm{r}=-0.469 ; \mathrm{P}=0.032)$.

Among all study subjects, abdominal aortic strain correlated significantly with $3 \mathrm{D}$ LA passive emptying volume/BSA $(\mathrm{r}=0.55 ; \mathrm{P}=0.001)$, LA passive contribution to $\mathrm{LV} \mathrm{SV}(\mathrm{r}=0.56 ; \mathrm{P}=0.001)$ and $3 \mathrm{D}$ LA total emptying volume/BSA $(\mathrm{r}=0.45 ; \mathrm{P}=0.003)$. 
Table 3 Characteristics, conventional Doppler and TDI Doppler in normal-weight and overweight/obese Turner syndrome patients (TS)

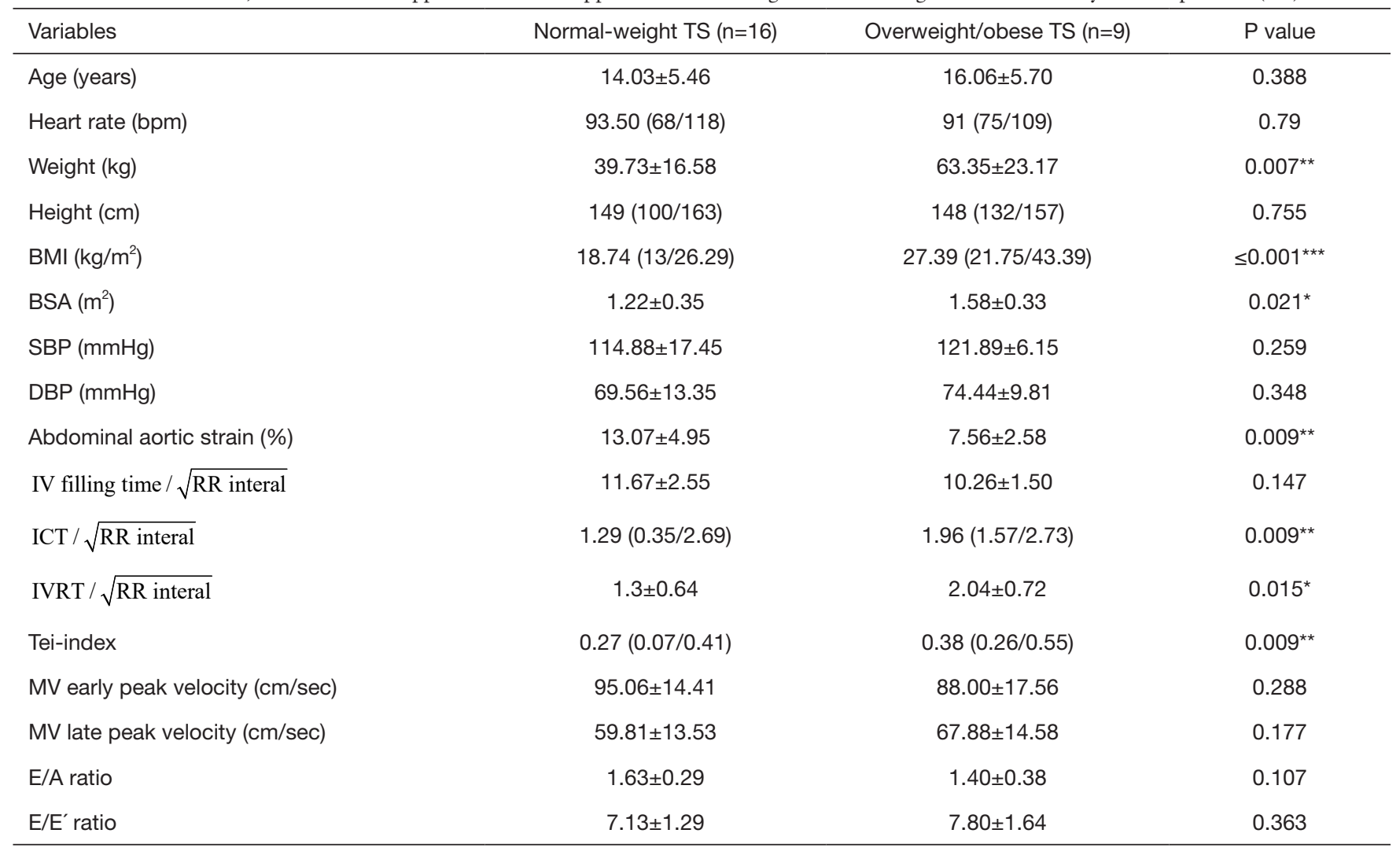

BMI, body mass index; BSA, body surface area; SBP, systolic brachial blood pressure; DBP, diastolic brachial blood pressure; ICT, isovolumetric contraction time; IVRT, isovolumetric relaxation time; E/E', pulsed MV early peak velocity to pulsed TDI (early diastolic myocardial velocities at the basal segment of the interventricular septum). Mean \pm standard deviation is used for normally distributed variables and median (minimum/maximum) for non-normally distributed variables. ${ }^{*} P$ value $\leq 0.05 ;{ }^{* *} P$ value $\leq 0.01 ;{ }^{* * *} P$ value $\leq 0.001$.

In a further analysis, TS patients were then divided into two groups according to the achieved median LA Vmax/ BSA [TS-group I ( $\mathrm{n}=16)$ : LA Vmax/BSA $\leq 18 \mathrm{~mL} / \mathrm{m}^{2}$; TSgroup II ( $\mathrm{n}=9)$ : LA Vmax/BSA $>18 \mathrm{~mL} / \mathrm{m}^{2}$ ]: No significant difference was assessed in age, weight, BMI, heart rate and BSA between both groups (Table 6). TS subjects with a LA Vmax/BSA $\leq 18 \mathrm{~mL} / \mathrm{m}^{2}$ displayed a significantly elevated SBP when compared to TS patients with a LA V $\max >18 \mathrm{~mL} / \mathrm{m}^{2}$ (121.69 15.49 vs. $109.78 \pm 9.62 \mathrm{mmHg} ; \mathrm{P}=0.049)$. Among all TS patients, SBP correlated significantly with 3D LA Vmax/BSA ( $\mathrm{r}=-0.49 ; \mathrm{P}=0.011)$, 3D LA passive emptying volume/BSA $(\mathrm{r}=-0.53 ; \mathrm{P}=0.006)$ and $3 \mathrm{D}$ LA total emptying volume/BSA $(r=-0.55 ; \mathrm{P}=0.004)$. Figure 3 visualises the relationship between 3D assessed LA volumes and the SBP within the TS cohort.

\section{Intraobserver variability}

Mean relative intraobserver variability was $0.36 \% \pm 6.03 \%$ for LA Vmin, $-0.56 \% \pm 4.60 \%$ for LA Vmax, $-0.42 \% \pm 5.44 \%$ for LA VpreA, $-0.72 \% \pm 4.11 \%$ for $3 \mathrm{D}$ LASr, $-0.50 \% \pm 8.28 \%$ for $3 \mathrm{D}$ LAScd, $-3.82 \% \pm 16.45 \%$ for 3D LASct, $5.28 \% \pm 16.87 \%$ for 3D LASr_c, $7.42 \% \pm 12.89 \%$ for 3D LAScd_c and $-4.02 \% \pm 37.58 \%$ for 3D LASct_c.

\section{Discussion}

To the best of our knowledge, this is the first study to investigate LA volumes and deformations in a cohort of TS patients. In total, 25 TS patients without CHD and 19 healthy, age-matched subjects were included for this study. LV and LA performance were evaluated through modern 
Table 4 Left atrial (LA) volumes and left ventricular (LV) volumes assessed using 3D echocardiography in healthy controls (HC) and normalweight Turner syndrome patients (TS)

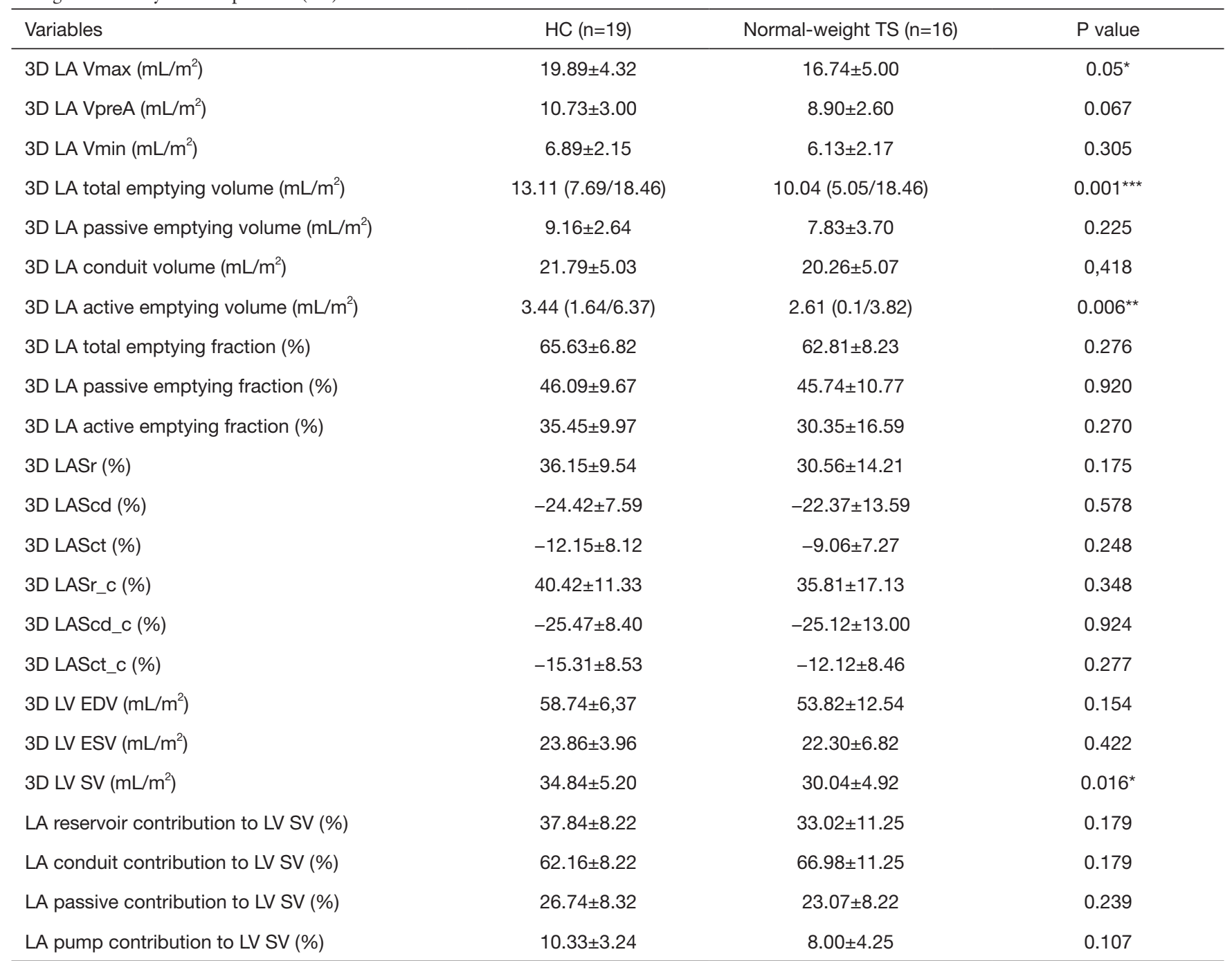

3D, three-dimensional echocardiography; LA Vmax, maximum LA volume at LV end systole; LA VpreA, LA volume at onset of LA contraction; LA Vmin, minimum LA volume at LV end diastole; LASr, longitudinal LA strain during reservoir phase; LAScd, longitudinal LA strain during conduit phase; LASct, longitudinal LA strain during contraction phase; LASr_c, circumferential LA strain during reservoir phase; LAScd_c, circumferential LA strain during conduit phase; LASct_c, circumferential LA strain during contraction phase; LV EDV, LV enddiastolic volume; LV ESV, LV endsystolic volume; LV SV, LV stroke volume. Mean \pm standard deviation is used for normally distributed variables and median (minimum/maximum) for non-normally distributed variables. ${ }^{\star} \mathrm{P}$ value $\leq 0.05 ;{ }^{* \star} \mathrm{P}$ value $\leq 0.01$; ${ }^{\star \star \star} \mathrm{P}$ value $\leq 0.001$.

techniques, including TDI Doppler, 2DSTE and 3DE.

\section{Conventional and TDI Doppler in TS patients and bealthy controls}

In line with other studies $(23,24)$, we observed a significantly increased heart rate in the examined TS patients. This phenomenon is assumed to be caused by sympathetic dysregulation (23). The data of the present study suggest that the LV filling time is the first-time interval to suffer from an increased heart rate in TS patients. Interestingly, the LV filling time of normal-weight TS subjects was, compared to healthy controls, reduced, even in the absence of manifested diastolic dysfunction (Table 2). Overweight/ 
Table 5 Left atrial (LA) volumes and left ventricular (LV) volumes assessed using 3D echocardiography in normal-weight and overweight/obese Turner syndrome patients (TS)

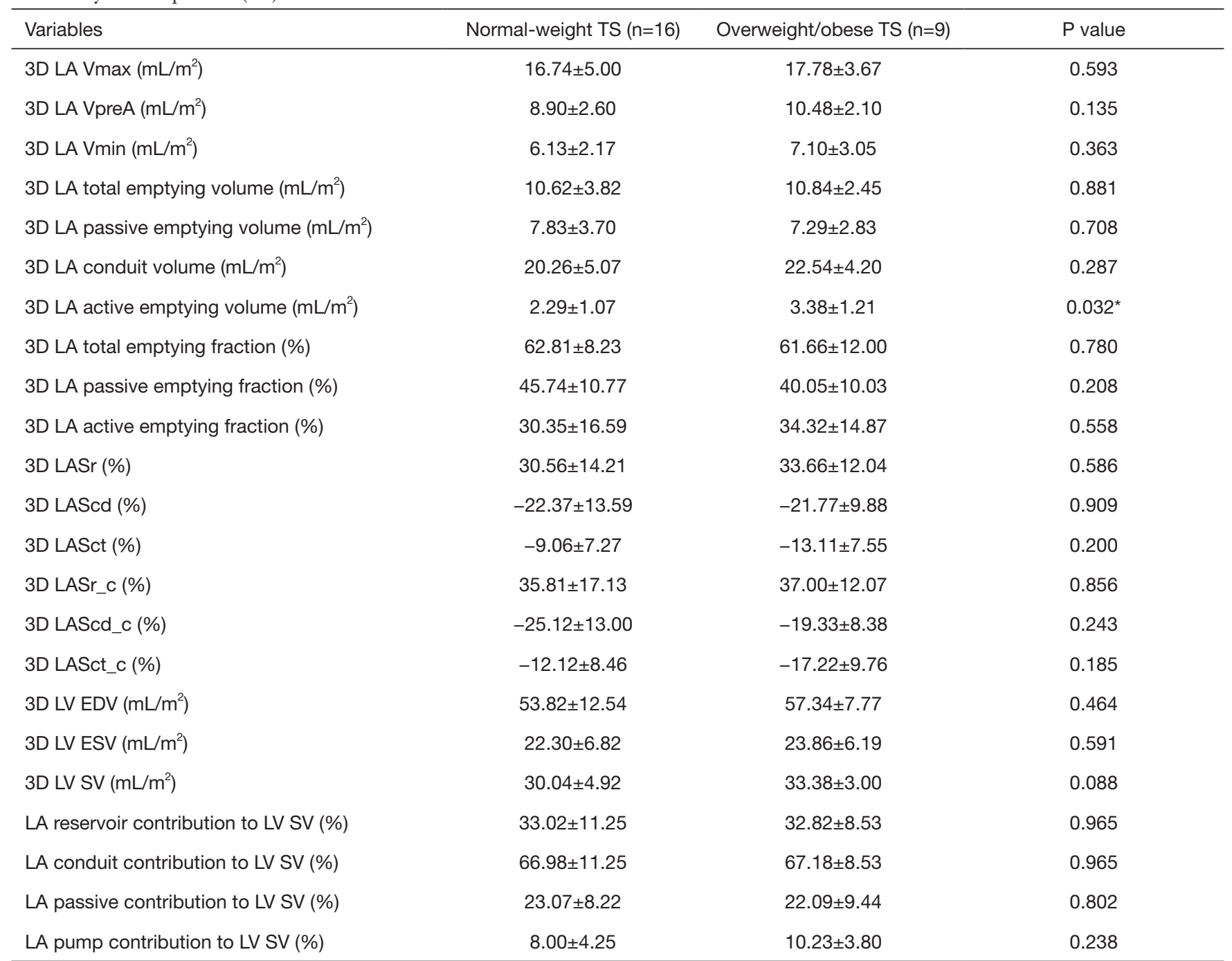

3D, three-dimensional echocardiography; LA Vmax, maximum LA volume at LV end systole; LA V preA, LA volume at onset of LA contraction; LA Vmin, minimum LA volume at LV end diastole; LASr, longitudinal LA strain during reservoir phase; LAScd, longitudinal LA strain during conduit phase; LASct, longitudinal LA strain during contraction phase; LASr_c, circumferential LA strain during reservoir phase; LAScd_c, circumferential LA strain during conduit phase; LASct_c, circumferential LA strain during contraction phase; LV EDV, LV enddiastolic volume; LV ESV, LV endsystolic volume; LV SV, LV stroke volume. Mean \pm standard deviation is used for normally distributed variables and median (minimum/maximum) for non-normally distributed variables. ${ }^{*} P$ value $\leq 0.05$.

obese TS patients displayed a significantly reduced LV myocardial performance when compared to normalweight TS patients. This was demonstrated by a significant prolongation of the isovolumetric contraction and relaxation time even in the presence of an increased heart rate.

TS is associated with an elevated arterial stiffness (5-8). In addition, a recent study of our department was able to show an increased LV afterload among TS patients (10). An augmentation in $L V$ afterload due to vascular dysfunction, might explain the abnormal LV relaxation and latent LV systolic dysfunction among TS subjects shown in the present study. An elevated heart rate might further aggravate these alterations. Funny channel inhibitors (e.g., ivabradine) are considered to improve myocardial contraction-relaxation coupling through normalization of isovolumic contraction and relaxation as well as heart rate independent mechanisms (25).

Further studies are required to investigate whether TS 


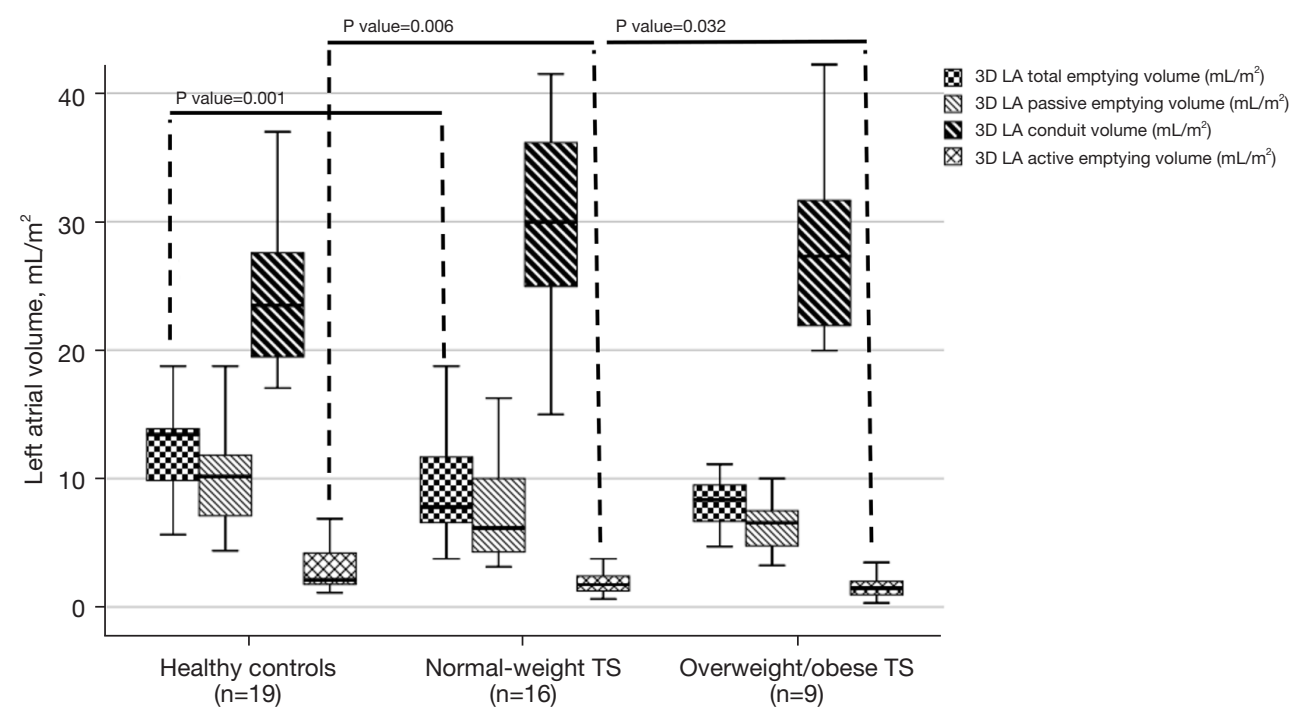

Figure 2 Left atrial (LA) emptying volumes assessed using three-dimensional (3D) echocardiography in healthy controls, normal-weight Turner syndrome (TS) patients and overweight/obese Turner syndrome patients (TS).

Table 6 Clinical characteristics of TS patients with a LA Vmax $\leq 18$ and $>18 \mathrm{~mL} / \mathrm{m}^{2}$

\begin{tabular}{lccc}
\hline Variables & TS LA Vmax $\leq 18 \mathrm{~mL} / \mathrm{m}^{2}(\mathrm{n}=16)$ & TS LA Vmax $>18 \mathrm{~mL} / \mathrm{m}^{2}(\mathrm{n}=9)$ & $\mathrm{P}$ value \\
\hline Age $($ years $)$ & $14.19 \pm 5.16$ & $15.77 \pm 6.29$ & 0.504 \\
Heart rate $(\mathrm{bpm})$ & $92.5(68 / 118)$ & $91(73 / 116)$ & 0.777 \\
Height $(\mathrm{cm})$ & $149(100 / 163)$ & $148(109 / 162)$ & 0.821 \\
Weight $(\mathrm{kg})$ & $49.33 \pm 23.78$ & $46.27 \pm 19.67$ & 0.746 \\
BMI $\left(\mathrm{kg} / \mathrm{m}^{2}\right)$ & $23.11 \pm 7.89$ & $22.19 \pm 5.33$ & 0.759 \\
BSA $\left(\mathrm{m}^{2}\right)$ & $1.37 \pm 0.39$ & $1.33 \pm 0.38$ & 0.801 \\
SBP $(\mathrm{mmHg})$ & $121.69 \pm 15.49$ & $109.78 \pm 9.62$ & $0.049^{\star}$ \\
DBP $(\mathrm{mmHg})$ & $73.69 \pm 13.09$ & $67.11 \pm 9.75$ & 0.203 \\
\hline
\end{tabular}

BMI, body mass index; BSA, body surface area; SBP, systolic brachial blood pressure; DBP, diastolic brachial blood pressure. Mean \pm standard deviation is used for normally distributed variables and median (minimum/maximum) for non-normally distributed variables. ${ }^{*} \mathrm{P}$ value $\leq 0.05$.

patients, especially those with excess weight, may benefit from heart rate-lowering medications such as beta blockers or funny channel inhibitors.

\section{Two-dimensional speckle tracking echocardiography and three-dimensional echocardiography for the assessment of left atrial size and function}

Under physiological conditions, LA reservoir and pump function increase with rising heart rate. The LA reservoir function comprises of the maintenance of atrioventricular diastolic pressure gradient depending on LV filling speed and the subsequent increase of LA pump function through preload mechanisms (26). In this study the LA total emptying volume/BSA, reflecting the atrial reservoir function, was significantly reduced among normalweight TS patients when compared to controls even with a significant raise in heart rate. Hence, the physiological LA reservoir function might be altered in normal-weight TS patients even without evident diastolic dysfunction. The reduced 3D LA Vmax/BSA is the main culprit of this finding, since the 3D Vmin/BSA did not differ significantly 


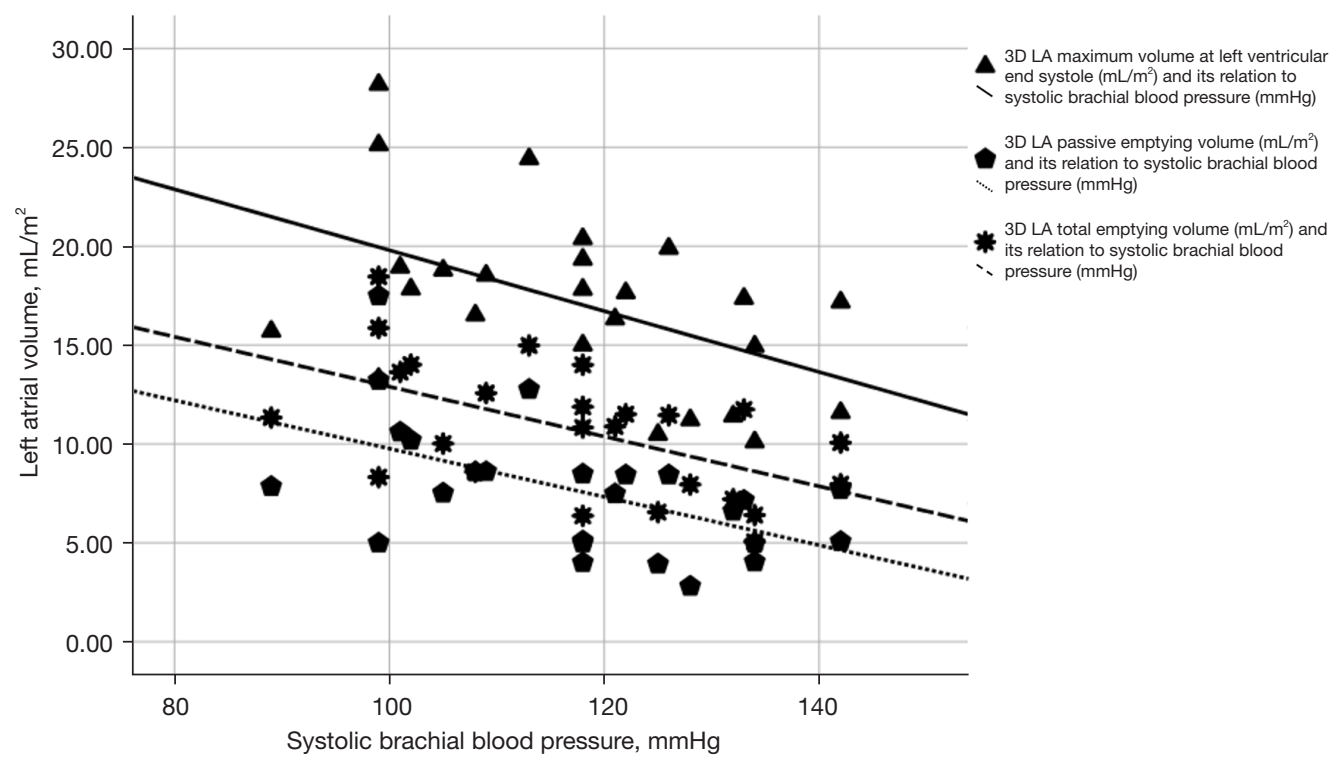

Figure 3 Relationship between left atrial (LA) volumes assessed using three-dimensional echocardiography and systolic brachial blood pressure within the Turner syndrome (TS) cohort.

between both groups. The 3D LA Vmax/BSA is mainly dependant on the LV systolic function (14). In a prior study of our department we were able to demonstrate a significantly reduced LV systolic function in TS subjects visualised by a significantly lower longitudinal LV Strain assessed using 3D speckle tracking echocardiography (10).

Based on the negative correlation between heart rate and the passive LA contribution to LV SV as well as the LA conduit longitudinal strain, a LV filling reduction among normal-weight TS patients may be presumed. This might explain the lower LV SV and consequently an increase in heart rate to maintain cardiac output (Table 4).

Overweight/obese TS patients demonstrated findings suggesting an abnormal LV relaxation pattern. The LA pump function was significantly increased among overweight/obese TS patients when compared to normalweight TS patients. This compensatory LA mechanism seems plausible for normal LV filling pressures. Invasive data reflecting the $\mathrm{LV}$ end diastolic pressure was not obtained in the present study and can be considered as a limitation. However, the E/E' ratio, a sensitive noninvasive marker of $L V$ end diastolic pressure, did not differ significantly between both groups.

In the present study, a LA Vmax/BSA $\leq 18 \mathrm{~mL} / \mathrm{m}^{2}$ was associated with a higher SBP within the TS cohort. These observations seem to be paradox. Previous reports in adult subjects showed a positive correlation between arterial blood pressure and LA size. Furthermore, LA size is considered to be a cardiovascular risk marker among adults with arterial hypertension (27). In the present study, the included TS patients were mainly of young age and without a history of arterial hypertension. This might partially explain the demonstrated results and suggest LA systolic expansion may not be a sensitive enough marker to reflect elevated arterial blood pressure in young TS subjects. Further studies with 24-hour ambulatory blood pressure monitoring are required to further elaborate these findings.

\section{Limitations}

\section{Study cohort and study design}

The relatively small number of patients included in the present study can be considered as a limitation. However, TS is a relatively rare disease affecting 1 in 2,500-3,000 female newborns. Furthermore, 13 TS subjects displaying CHD were excluded from this study for better data comparability. In literature CHD is reported to affect up to $50 \%$ of TS patients (4). Regarding these aspects, we consider a cohort of 25 TS patients without CHD to be adequate. Nonetheless, longitudinal multi-center studies are required to better understand cardiovascular morbidity of TS, including the potential effects of growth hormone therapy on increasing myocardial mass and its role in LA and LV function preservation. 
Transthoracic echocardiography versus cardiac magnetic resonance imaging

The assessment of LA function through 2DSTE and 3DE can be limited by suboptimal echocardiographic window leading to poor image quality. Considering that TS is often associated with excess weight, cardiac magnetic resonance imaging (cMRI) might offer a superior imaging modality in the assessment of LA function especially in adult subjects. However, in pediatric TS patients, sedation is often required to carry out cMRI, making this method inapplicable in the routine outpatient care. In addition, current cMRI myocardial tagging techniques have rather low spatial resolution. In contrast, high spatial resolution images suffer from low signal noise ratio. Accordingly, this technique might not be applicable in LA function assessment of TS patients presenting with increased heart rate.

\section{Conclusions}

Normal-weight TS patients with relatively increased heart rate and reduced LV filling time display subtle LV diastolic dysfunction in the form of reduced LA reservoir and pump function. Manifested systolic and diastolic LV dysfunction among overweight TS patients is partially compensated through an increase in their LA active pump function.

\section{Acknowledgments}

We would like to thank all study participants and the German Turner Syndrome Association (Turner-SyndromVereinigung Deutschland e.V.) for the interest in our study. We thank Megan Crouse for editorial assistance.

Funding: This study was supported by the Competence Network for Congenital Heart Defects, which received funding from the Federal Ministry of Education and Research [01GI0601 (2014)], and the German Centre for Cardiovascular Research [81X2800112 (2015)].

\section{Footnote}

Reporting Checklist: The authors have completed the STROBE reporting checklist. Available at https://qims. amegroups.com/article/view/10.21037/qims-21-515/rc

Conflicts of Interest: All authors have completed the ICMJE uniform disclosure form (available at https://qims. amegroups.com/article/view/10.21037/qims-21-515/coif). The authors have no conflicts of interest to declare.
Ethical Statement: The authors are accountable for all aspects of the work in ensuring that questions related to the accuracy or integrity of any part of the work are appropriately investigated and resolved. This study was conducted in accordance with the Declaration of Helsinki (as revised in 2013). This study was approved by the Ethics Committee of the Ärztekammer des Saarlandes (State Chamber of Physicians of the German federal state of Saarland), Faktoreistraße 4, 66111 Saarbrücken, Germany, on March 23rd, 2018; approval statement No. 07/18. Prior written informed consent was obtained from all patients or the parents or legal guardians of patients under legal age.

Open Access Statement: This is an Open Access article distributed in accordance with the Creative Commons Attribution-NonCommercial-NoDerivs 4.0 International License (CC BY-NC-ND 4.0), which permits the noncommercial replication and distribution of the article with the strict proviso that no changes or edits are made and the original work is properly cited (including links to both the formal publication through the relevant DOI and the license). See: https://creativecommons.org/licenses/by-nc-nd/4.0/.

\section{References}

1. Sybert VP, McCauley E. Turner's syndrome. N Engl J Med 2004;351:1227-38.

2. Schoemaker MJ, Swerdlow AJ, Higgins CD, Wright AF, Jacobs PA; United Kingdom Clinical Cytogenetics Group. Mortality in women with turner syndrome in Great Britain: a national cohort study. J Clin Endocrinol Metab 2008;93:4735-42.

3. Mavinkurve $\mathrm{M}, \mathrm{O}^{\prime}$ Gorman CS. Cardiometabolic and vascular risks in young and adolescent girls with Turner syndrome. BBA Clin 2015;3:304-9.

4. Gravholt CH, Andersen NH, Conway GS, Dekkers OM, Geffner ME, Klein KO, Lin AE, Mauras N, Quigley CA, Rubin K, Sandberg DE, Sas TCJ, Silberbach M, Söderström-Anttila V, Stochholm K, van Alfen-van derVelden JA, Woelfle J, Backeljauw PF; International Turner Syndrome Consensus Group. Clinical practice guidelines for the care of girls and women with Turner syndrome: proceedings from the 2016 Cincinnati International Turner Syndrome Meeting. Eur J Endocrinol 2017;177:G1-G70.

5. An HS, Baek JS, Kim GB, Lee YA, Song MK, Kwon BS, Bae EJ, Noh CI. Impaired Vascular Function of the Aorta in Adolescents with Turner Syndrome. Pediatr Cardiol 
2017;38:20-6.

6. Uçar A, Öz F, Baş F, Oflaz H, Nişli K, Tuğrul M, Yetim A, Darendeliler F, Saka N, Poyrazoğlu Ş, Bundak R. Increased arterial stiffness in young normotensive patients with Turner syndrome: associations with vascular biomarkers. Clin Endocrinol (Oxf) 2015;82:719-27.

7. De Groote K, Devos D, Van Herck K, De Wolf D, Van der Straaten S, Rietzschel E, Raes A, Vandekerckhove K, Panzer J, De Wilde H, De Schepper J. Increased aortic stiffness in prepubertal girls with Turner syndrome. J Cardiol 2017;69:201-7.

8. Oberhoffer FS, Abdul-Khaliq H, Jung AM, Rohrer TR, Abd El Rahman M. Two-dimensional speckle tracking of the abdominal aorta: a novel approach to evaluate arterial stiffness in patients with Turner syndrome. Cardiovasc Diagn Ther 2019;9:S228-37.

9. Oberhoffer FS, Abdul-Khaliq H, Jung AM, Zemlin M, Rohrer TR, Abd El Rahman M. Assessment of left ventricular myocardial work in Turner syndrome patients: insights from the novel non-invasive pressurestrain loop analysis method. Quant Imaging Med Surg 2020;10:15-25.

10. Oberhoffer FS, Abdul-Khaliq H, Jung AM, Rohrer TR, Abd El Rahman M. Left ventricular remodelling among Turner syndrome patients: insights from non-invasive 3D echocardiography-derived pressure-volume loop analysis. Clin Res Cardiol 2020;109:892-903.

11. Oberhoffer FS, Abdul-Khaliq H, Jung M, Rohrer R, Abd El Rahman M. Left Ventricular Diastolic Dysfunction in Patients with Turner's Syndrome. Thorac Cardiovasc Surg 2019;67:S101-S128.

12. Piotrowski G, Goch A, Wlazłowski R, Gawor Z, Goch $\mathrm{JH}$. Non-invasive methods of atrial function evaluation in heart diseases. Med Sci Monit 2000;6:827-39.

13. Prioli A, Marino P, Lanzoni L, Zardini P. Increasing degrees of left ventricular filling impairment modulate left atrial function in humans. Am J Cardiol 1998;82:756-61.

14. Cameli M, Lisi M, Focardi M, Reccia R, Natali BM, Sparla $\mathrm{S}$, Mondillo S. Left atrial deformation analysis by speckle tracking echocardiography for prediction of cardiovascular outcomes. Am J Cardiol 2012;110:264-9.

15. Abd El Rahman M, Raedle-Hurst T, Rentzsch A, Schäfers HJ, Abdul-Khaliq H. Assessment of inter-atrial, inter-ventricular, and atrio-ventricular interactions in tetralogy of Fallot patients after surgical correction. Insights from two-dimensional speckle tracking and three-dimensional echocardiography. Cardiol Young 2015;25:1254-62.
16. Kromeyer-Hauschild K, Wabitsch M, Kunze D, Geller F, Geiß HC, Hesse V, et al. Perzentile für den Body-massIndex für das Kindes- und Jugendalter unter Heranziehung verschiedener deutscher Stichproben. Monatsschr Kinderheilkd 2001;149:807-18.

17. Lopez L, Colan SD, Frommelt PC, Ensing GJ, Kendall K, Younoszai AK, Lai WW, Geva T. Recommendations for quantification methods during the performance of a pediatric echocardiogram: a report from the Pediatric Measurements Writing Group of the American Society of Echocardiography Pediatric and Congenital Heart Disease Council. J Am Soc Echocardiogr 2010;23:465-95; quiz 576-7.

18. Tei C, Dujardin KS, Hodge DO, Kyle RA, Tajik AJ, Seward JB. Doppler index combining systolic and diastolic myocardial performance: clinical value in cardiac amyloidosis. J Am Coll Cardiol 1996;28:658-64.

19. Abd El Rahman MY, Abdul-Khaliq H, Vogel M, AlexiMeskischvili V, Gutberlet M, Hetzer R, Lange PE. Value of the new Doppler-derived myocardial performance index for the evaluation of right and left ventricular function following repair of tetralogy of fallot. Pediatr Cardiol 2002;23:502-7.

20. Mosteller RD. Simplified calculation of body-surface area. N Engl J Med 1987;317:1098.

21. Chen L, Zhang C, Wang J, Guo L, Wang X, Liu F, Li X, Zhao Y. Left atrial strain measured by 4D Auto LAQ echocardiography is significantly correlated with high risk of thromboembolism in patients with nonvalvular atrial fibrillation. Quant Imaging Med Surg 2021;11:3920-31.

22. Blume GG, Mcleod CJ, Barnes ME, Seward JB, Pellikka PA, Bastiansen PM, Tsang TS. Left atrial function: physiology, assessment, and clinical implications. Eur J Echocardiogr 2011;12:421-30.

23. Zuckerman-Levin N, Zinder O, Greenberg A, Levin M, Jacob G, Hochberg Z. Physiological and catecholamine response to sympathetic stimulation in turner syndrome. Clin Endocrinol (Oxf) 2006;64:410-5.

24. Tancredi G, Versacci P, Pasquino AM, Vittucci AC, Pucarelli I, Cappa M, Di Mambro C, Marino B. Cardiopulmonary response to exercise and cardiac assessment in patients with turner syndrome. Am J Cardiol 2011;107:1076-82.

25. Melka J, Rienzo M, Bizé A, Jozwiak M, Sambin L, Hittinger L, Su JB, Berdeaux A, Ghaleh B. Improvement of left ventricular filling by ivabradine during chronic hypertension: involvement of contraction-relaxation 
coupling. Basic Res Cardiol 2016;111:30.

26. Nishikawa Y, Roberts JP, Tan P, Klopfenstein CE, Klopfenstein HS. Effect of dynamic exercise on left atrial function in conscious dogs. J Physiol 1994;481:457-68.

Cite this article as: Abd El Rahman M, Jung AM, Zemlin M, Rohrer TR, Schuck R, Oberhoffer FS, Abdul-Khaliq H. Left atrial remodelling among Turner syndrome patients: novel insights from non-invasive 3D echocardiography. Quant Imaging Med Surg 2022;12(5):2634-2648. doi: 10.21037/qims21-515
27. Eshoo S, Ross DL, Thomas L. Impact of mild hypertension on left atrial size and function. Circ Cardiovasc Imaging 2009;2:93-9. 